\title{
Relationship between plasma and brain levels and the anticonvulsant effect of lamotrigine in rats
}

\author{
Margarida Castel-Branco, Vera Lebre, Amílcar Falcão*, Isabel Figueiredo, Margarida Caramona \\ Laboratory of Pharmacology, Faculty of Pharmacy, Coimbra University, 3000-295 Coimbra, Portugal
}

Received 30 June 2003; received in revised form 24 September 2003; accepted 30 September 2003

\begin{abstract}
The aims of the present research were to characterize the plasma and brain concentration-time profiles of lamotrigine after its acute administration and to establish the relationship between these profiles and the anticonvulsant effect of lamotrigine in rats. Adult male Wistar rats were given $10 \mathrm{mg} / \mathrm{kg}$ of lamotrigine intraperitoneally. The lamotrigine profiles in plasma and brain were determined at predetermined times over 120 -h post-dose. The anticonvulsant profile against maximal electroshock seizure stimulation was determined over $48 \mathrm{~h}$ after dosing. After injection, lamotrigine rapidly appeared in both plasma and brain, suggesting ready penetration of the blood-brain barrier. A linear relationship was established between lamotrigine plasma and brain levels. A linear relationship was also defined between lamotrigine brain levels and the anticonvulsant response. The correspondent plasma levels were also well correlated with the anticonvulsant effect from the moment that the plasma-brain system had reached equilibrium.
\end{abstract}

(C) 2003 Elsevier B.V. All rights reserved.

Keywords: Lamotrigine; Anticonvulsant; Electroshock, maximal; Pharmacokinetics; Pharmacodynamics

\section{Introduction}

Lamotrigine (3,5-diamino-6-(2,3-dichlorophenyl)-1,2,4triazine) is a new generation antiepileptic drug which has been shown to be effective against partial and secondarily generalized tonic-clonic seizures, either as adjunctive treatment in patients with refractory epilepsy or when received as monotherapy (Goa et al., 1993). Actually, lamotrigine exhibits a relatively broad spectrum of efficacy, against some common seizure types, such as partial seizures (with or without secondary generalization), primarily generalized tonic-clonic seizures, absence seizures and drop attacks. Its efficacy in myoclonic seizures and infantile spasms is still unclear (Perucca, 2002).

The first mechanism of action of lamotrigine referred to in the literature was similar to that proposed for carbamazepine and phenytoin and involved the stabilization of the presynaptic membrane through the blockade of the voltagesensitive $\mathrm{Na}^{+}$channels, which resulted in the inhibition of excitatory neurotransmitter release, particularly glutamate

\footnotetext{
* Corresponding author. Tel.: +351-239-820510; fax: +351-239837731.

E-mail address: acfalcao@ff.uc.pt (A. Falcão).
}

and aspartate (Goa et al., 1993). More recently, it was proposed that lamotrigine also inhibits high voltage-activated $\mathrm{Ca}^{2+}$ currents, interacting consequently with the vesicular release of transmitters (Waldmeier et al., 1995; Stefani et al., 1996).

The use of lamotrigine in clinical practice is reasonably well tolerated by patients (Richens, 1994). However, the resulting benefits of its administration are subject to a more complex evaluation, given the considerable inter-patient variability observed in relation to the dosage required to obtain an appropriate therapeutic response. A notional target range of $1-4 \mathrm{mg} / \mathrm{l}$ was initially proposed, but subsequent observations have indicated that some patients may tolerate much higher therapeutic concentrations $(>10 \mathrm{mg} / \mathrm{l})$ without clinical toxicity. A linear relationship appears to exist between the doses of lamotrigine administered and the respective plasma concentrations. Thus, the difficulty lies in the establishment of a relationship between lamotrigine plasma levels and the induced pharmacological response. In the light of current knowledge, it seems evident that, to assess a relationship between plasma concentration and clinical effect of lamotrigine, further studies are required (Kilpatrick et al., 1996; Bartoli et al., 1997; Morris et al., 1998).

As with all antiepileptics, lamotrigine needs to cross the blood-brain barrier to exert its therapeutic effect. 
Consequently, the interpretation of the lamotrigine plasma levels requires the assessment of the drug concentration at the neuronal sites of action. Afterwards, the characterization of the experimental anticonvulsant profile of lamotrigine is important in the evaluation of its pharmacological response. Accordingly, the aims of the present research were to define the plasma and brain concentration-time profiles of lamotrigine after its administration as a single intraperitoneal dose and to establish the relationship between these profiles and the anticonvulsant effect of lamotrigine against maximal electroshock seizures in rats. The analysis of the results obtained from these studies is thought to bring some light to the assessment of the relationship between plasma concentration and clinical effect of lamotrigine.

\section{Materials and methods}

\subsection{Animals}

The studies were carried out on adult male Wistar rats, weighing 250-320 g (Harlan Iberica, Barcelona, Spain). The rats were housed in a local bioterium with a controlled 12-h light/dark cycle. Animals were allowed free access to food and water until the experiments, performed at $22-23{ }^{\circ} \mathrm{C}$. Animal experimentation in this study was conducted in accordance with the European guidelines for the care and use of laboratory animals (86/ $609 /$ EEC) and the project was approved by the Portuguese Veterinary General Division.

\subsection{Drugs}

Lamotrigine, lamotrigine isethionate and the internal standard (3,5-diamino-6-(2-methoxyphenyl)-1,2,4-triazine (BW725C78) were kindly provided by Wellcome Research Laboratories (Cardiff, UK). Ketamine hydrochloride $(7.7 \mathrm{mg} / \mathrm{kg}$ ) (Pfizer Laboratories, Seixal, Portugal) and chlorpromazine $(2.3 \mathrm{mg} / \mathrm{kg})$ (Vitória Laboratories, Amadora, Portugal) were used to anaesthetise the animals before sample collection. Oxibuprocaine chloride (Oftalder, Oeiras, Portugal) was used to anaesthetise the rats' eyes. Reagents and columns used in the chromatographic analysis were purchased from Merck (Darmstadt, Germany).

\subsection{Experimental design}

For the characterization of systemic and central lamotrigine profiles in the rat, a group of 45 animals was given $10 \mathrm{mg} / \mathrm{kg}$ of lamotrigine intraperitoneally in an aqueous solution of lamotrigine isethionate (Castel-Branco et al., 2002). Sample collection occurred at predetermined times. Subgroups of five animals were used at each data point. Blood samples were obtained by open cardiac puncture and collected in citrated tubes at 7.5, 15 and 30 min, 2-, 12-, 24-, 48-, 72- and 120-h post-dose. Immediately afterwards, the animals were decapitated and the brains removed to be homogenized in $5 \mathrm{ml}$ of phosphate buffer $(\mathrm{pH}=7.4)$ per $\mathrm{g}$ of tissue at $4{ }^{\circ} \mathrm{C}$. Blood collection was carried out under anaesthesia injected intramuscularly $10 \mathrm{~min}$ before the referred to procedure. Plasma and whole brain homogenates were immediately frozen at $-25{ }^{\circ} \mathrm{C}$ until analysis.

For the assessment of the anticonvulsant profile of lamotrigine in the rat following a $10 \mathrm{mg} / \mathrm{kg}$ intraperitoneal dose, a second group of animals was used. Maximal electroshock seizure stimulation was applied through bipolar corneal electrodes from an electroshock apparatus (Ugo Basile ECT unit 7801) at an intensity sufficient to elicit tonic hindlimb extension in $\sim 100 \%$ of the control animals (current: $150 \mathrm{~mA}$; frequency: $60 \mathrm{~Hz}$; pulse width: $0.6 \mathrm{~ms}$; duration: $0.2 \mathrm{~s}$ ) (Löscher et al., 1991). Immediately before placement of the electrodes, a drop of the anaesthetic oxibuprocaine chloride was introduced into the rat eyes. Only the animals that consistently exhibited the tonic hindlimb extensor component of maximal electroshock seizure during three trials on separate days, while unmedicated, were used (Swinyard et al., 1952; Browning and Nelson, 1985; Ramzan and Levy, 1989). Eight animals per group were tested once at a predetermined time after drug injection: 15 and 30 min, 1-, 2-, 4-, 8-, 12-, 24-, 36- and 48-h post-dose. The anticonvulsant activity of lamotrigine was determined as a quantal endpoint (i.e. the presence or absence of tonic hindlimb extension). The number of animals protected against maximal electroshock seizure stimulation was converted into a percentage (eight animals protected $=100 \%$ protected).

\subsection{Lamotrigine quantification}

Lamotrigine levels in plasma and brain homogenate were determined according to a high-performance liquid chromatography (HPLC) method, previously described (Castel-Branco et al., 2001). Briefly, to $1 \mathrm{ml}$ of plasma, $100 \mu \mathrm{l}$ of a $40 \mathrm{mg} / \mathrm{l}$ internal standard solution, $1 \mathrm{ml}$ of 2 $\mathrm{M} \mathrm{NaOH}$ and $5 \mathrm{ml}$ of ethyl acetate were added. After centrifugation, the upper organic layer was transferred to a clean $10-\mathrm{ml}$ conical glass tube and evaporated to dryness. The brain homogenate extraction included a previous deproteinization step: to $1 \mathrm{ml}$ of brain homogenate, $100 \mu \mathrm{l}$ of a $20 \mathrm{mg} / \mathrm{l}$ internal standard solution and $100 \mu \mathrm{l}$ of a $20 \%$ trichloroacetic acid solution were added. After centrifugation, the supernatant was transferred to a $10-\mathrm{ml}$ glass tube and submitted to a liquid-liquid extraction into ethyl acetate after basification, as described for plasma. The residues obtained were reconstituted with $200 \mu \mathrm{l}$ of mobile phase and injected into the HPLC system. Chromatographic separation was carried out on a LiChrospher 100 RP-18 (5 $\mu \mathrm{m})$ LiChroCART 125-4 
(Merck) for $10 \mathrm{~min}$. The mobile phase, consisting of $35.0 \%$ methanol, $64.7 \% \quad 0.1 \mathrm{M}$ potassium dihydrogen phosphate solution and $0.3 \%$ triethylamine, was pumped at a flow rate of $1.0 \mathrm{ml} / \mathrm{min}$. The detector was set at 306 $\mathrm{nm}$. The linearity was demonstrated over a range of $0.1-$ $15.0 \mathrm{mg} / \mathrm{l}$ for plasma and $0.1-5.0 \mathrm{mg} / \mathrm{l}$ for brain homogenate, with a lamotrigine detection limit of 0.01 and 0.02 $\mathrm{mg} / \mathrm{l}$ in plasma and brain homogenate, respectively. The results of the method validation were all in accordance with international recommendations, providing the suitability of the method for lamotrigine quantification in these biological matrices.

\section{Results}

\subsection{Lamotrigine plasma and brain homogenate concen- tration-time profiles}

Plasma and brain homogenate concentrations of lamotrigine following a single $10 \mathrm{mg} / \mathrm{kg}$ intraperitoneal dose are presented in Table 1. The experimental peak plasma value was considered achieved at 15-min post-dose, despite the fact that no major differences had been found between the plasma levels measured at 15, $30 \mathrm{~min}$ and 2-h post-dose (analysis of variance, $P>0.05$ ). Brain homogenate experimental concentrations peaked somewhat later than plasma levels, between $30 \mathrm{~min}$ and $2 \mathrm{~h}$ after the intraperitoneal injection. After peak values, both lamotrigine plasma and brain levels decreased exponentially. The lamotrigine brain homogenate levels determined at 120 -h post-dose were not

Table 1

Plasma and brain homogenate concentrations of lamotrigine and its anticonvulsant effect against maximal electroshock seizures following a single $10 \mathrm{mg} / \mathrm{kg}$ intraperitoneal dose of lamotrigine in the rat

\begin{tabular}{|c|c|c|c|c|c|}
\hline \multirow{2}{*}{$\begin{array}{l}\text { Time after } \\
\text { administration (h) }\end{array}$} & \multicolumn{2}{|c|}{ Plasma levels (mg/l) } & \multicolumn{2}{|c|}{ Brain levels (mg/l) } & \multirow{2}{*}{$\begin{array}{l}\text { Protection } \\
(\%)^{\mathrm{a}}\end{array}$} \\
\hline & $C_{\text {experimental }} \mathrm{b}$ & $C_{\text {fitted }}{ }^{\mathrm{c}}$ & $\begin{array}{r}\mathrm{b} \\
C_{\text {experimental }}\end{array}$ & $C_{\text {fitted }}^{\mathrm{c}}$ & \\
\hline 0.125 & $2.98 \pm 0.364$ & 3.22 & $0.44 \pm 0.244$ & 0.51 & - \\
\hline 0.25 & $5.42 \pm 0.798$ & 4.36 & $1.02 \pm 0.301$ & 0.87 & 25.0 \\
\hline 0.5 & $4.99 \pm 1.099$ & 4.89 & $1.64 \pm 0.255$ & 1.29 & 62.5 \\
\hline 1 & - & 4.91 & - & 1.57 & 87.5 \\
\hline 2 & $4.83 \pm 0.916$ & 4.79 & $1.67 \pm 0.283$ & 1.63 & 100.0 \\
\hline 4 & - & 4.56 & - & 1.57 & 100.0 \\
\hline 8 & - & 4.13 & - & 1.45 & 87.5 \\
\hline 12 & $3.44 \pm 0.488$ & 3.73 & $1.21 \pm 0.205$ & 1.34 & 62.5 \\
\hline 24 & $2.98 \pm 0.545$ & 2.77 & $1.06 \pm 0.290$ & 1.05 & 12.5 \\
\hline 36 & - & 2.05 & - & 0.83 & 12.5 \\
\hline 48 & $1.29 \pm 0.373$ & 1.52 & $0.57 \pm 0.115$ & 0.65 & 0.0 \\
\hline 72 & $1.03 \pm 0.191$ & 0.83 & $0.44 \pm 0.156$ & 0.40 & - \\
\hline 120 & $0.25 \pm 0.133$ & 0.25 & - & 0.15 & - \\
\hline
\end{tabular}

\footnotetext{
${ }^{a}$ Percentage protection against maximal electroshock seizure stimulation (eight animals).

${ }^{\mathrm{b}}$ Mean \pm standard deviation (five animals).

${ }^{c}$ Concentration obtained from the biexponential equation used to fit the experimental values (Yamaoka et al., 1981): $C_{\text {plasma }}=5.039 \mathrm{e}^{-0.025 . t}$ $5.039 \mathrm{e}^{-8.203 . t}$ and $C_{\text {brain }}=1.699 \mathrm{e}^{-0.020 . t}-1.699 \mathrm{e}^{-2.908 . t}$.
}

considered because they were below the limit of quantification of the analytical technique $(0.1 \mathrm{mg} / \mathrm{l})$.

\subsection{Lamotrigine brain/plasma relationship}

After the absorption phase ( $\geq 0.5$-h post-dose), a linear correlation was established between the mean lamotrigine values determined in plasma and in brain homogenate, with $r^{2}=0.997$.

The degree of drug uptake from plasma into brain tissue was estimated from the ratio of the drug concentration in brain homogenate over the plasma concentration ( $\left.C_{\text {brain homogenate }} / C_{\text {plasma }}\right)$. The mean calculated ratio value from all measurements between 0.5 - and 72 -h post-dose was $0.38 \pm 0.097$ (mean \pm standard deviation). In order to make possible the comparison of this uptake between different experimental studies, the brain homogenate concentration values, expressed by $\mu \mathrm{g} / \mathrm{ml}$ ( $5 \mathrm{ml}$ of phosphate buffer per $\mathrm{g}$ of brain tissue), were converted into values expressed by $\mu \mathrm{g} /$ $\mathrm{g}$ of brain tissue. This conversion was performed by assuming that brain specific gravity is $1.0 \mathrm{~g} / \mathrm{ml}$ (Adusumalli et al., 1993; Deguchi et al., 1995). The mean calculated brain/ plasma concentration ratio value between 0.5 - and 72 -h postdose was, therefore, $2.29 \pm 0.580$.

\subsection{Lamotrigine anticonvulsant profile}

The anticonvulsant responses of lamotrigine evaluated over $48 \mathrm{~h}$ following a single $10 \mathrm{mg} / \mathrm{kg}$ intraperitoneal dose are presented in Table 1. Lamotrigine showed effectiveness within $15 \mathrm{~min}$ - the shortest time tested-with maximal anticonvulsant activity between 2- and 4-h post-dose. A very considerable protection $(>50 \%)$ persisted up to $12 \mathrm{~h}$, but at 48 -h post-dose no more protection was detected.

\subsection{Lamotrigine anticonvulsant effect-plasma and brain concentration relationships}

Time courses of lamotrigine plasma and brain homogenate concentrations and its anticonvulsant effect against maximal electroshock seizures are reported together in Fig. 1.

To define the kind of relationship established between the anticonvulsant effect and the matching brain concentrations, the values of the lamotrigine anticonvulsant responses were plotted against the corresponding lamotrigine brain levels, as shown in Fig. 2(B). The regression analysis performed established a linear relationship between the lamotrigine brain concentrations and the respective anticonvulsant effects, by revealing a coefficient of determination $\left(r^{2}\right)$ of 0.937 .

Relative to the effect-plasma concentration relationship, the regression analysis presented a $r^{2}$ of 0.684 (Fig. 2(A)). However, when the regression analysis was performed after rejecting the two first data points, a strong correlation was observed between lamotrigine plasma levels and the phar- 


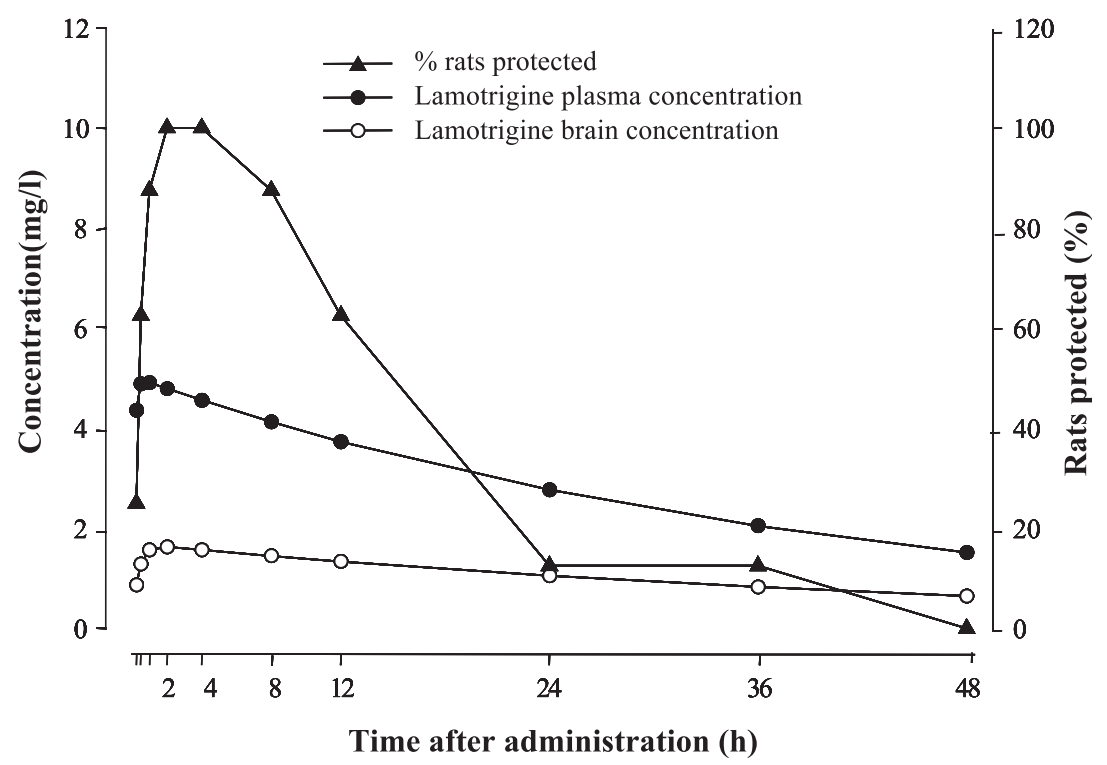

Fig. 1. Lamotrigine anticonvulsant effect against maximal electroshock seizures (right vertical axis) versus time profile and plasma and brain homogenate lamotrigine concentration (left vertical axis) versus time biexponential profiles following a single $10 \mathrm{mg} / \mathrm{kg}$ intraperitoneal dose of lamotrigine in the rat.

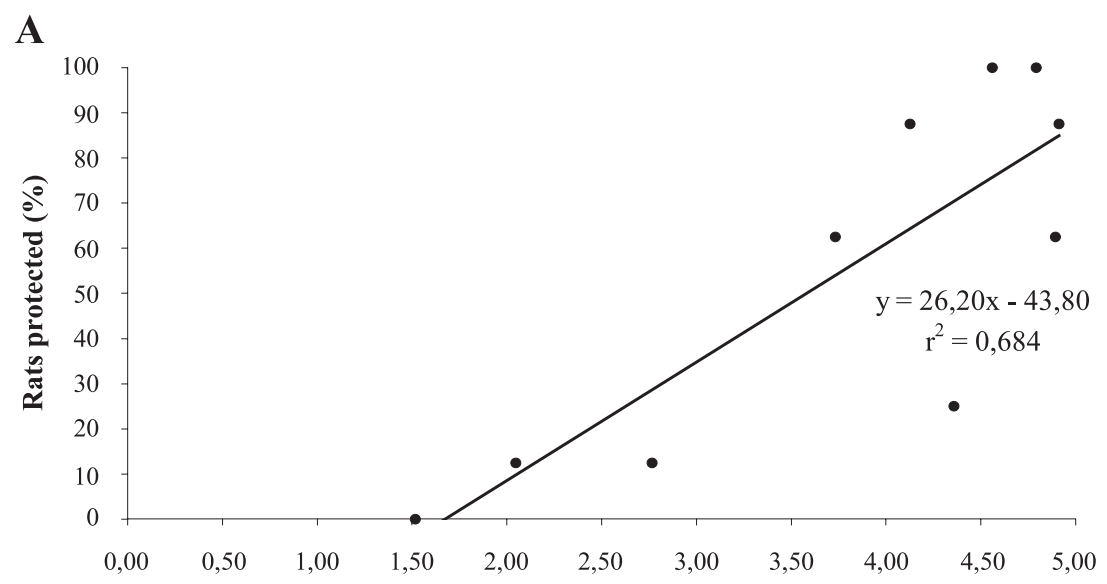

Lamotrigine plasma concentration (mg/l)

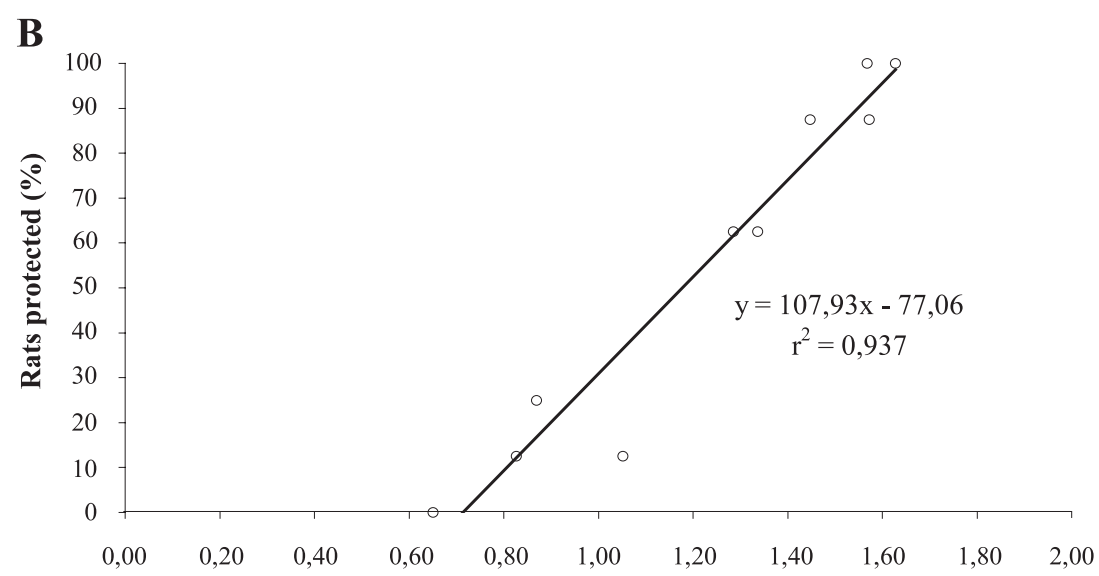

Lamotrigine brain homogenate concentration (mg/l)

Fig. 2. Anticonvulsant responses to lamotrigine, expressed as the $\%$ of rats protected, versus lamotrigine plasma levels (A) and lamotrigine brain homogenate levels (B) following a single $10 \mathrm{mg} / \mathrm{kg}$ intraperitoneal dose of lamotrigine in the rat (sampling time from 0.25 to $48 \mathrm{~h}$ after administration). 
macological response $\left(r^{2}=0.930\right.$; sampling time from 1 to $48 \mathrm{~h}$ after administration).

\section{Discussion}

The present study was designed and performed bearing in mind the difficulty that still currently exists in the establishment of a relationship between lamotrigine plasma levels and the induced pharmacological response. In spite of the microdialysis study carried out by Walker et al. (2000), the present data represent additional information for the characterization of lamotrigine neuropharmacokinetics in rats, since it is important to compare the extracellular with the total brain levels of the drug to define the pharmacologically relevant concentration. Additionally, as far as we are aware, the present research represents the first report on the temporal inter-relationship of lamotrigine plasma and brain concentrations and its anticonvulsant activity evaluated against maximal electroshock.

A dose of $10 \mathrm{mg} / \mathrm{kg}$ was chosen because this dose had previously been found to be within the anticonvulsive range in the rat (Wheatley and Miller, 1989). Also, with this dose, the plasma concentrations achieved were similar to the concentration range that had been proposed for epileptic patients (Morris et al., 1998).

After intraperitoneal administration, lamotrigine rapidly appeared in plasma (peak value at $0.25 \mathrm{~h}$ ), demonstrating rapid absorption from the peritoneal cavity. In addition, lamotrigine quickly appeared in the brain (peak value between 0.5 and $2 \mathrm{~h}$ ), suggesting ready penetration of the blood-brain barrier. These observations are comparable to those previously reported by Walton et al. (1996) and Walker et al. (2000). After peak values, a monoexponential fall was observed both in lamotrigine plasma and brain concentrations. Taking into account the parallel patterns observed in lamotrigine plasma and brain profiles - demonstrated in this study by the linear relationship established between drug in plasma and drug in brain-it can be assumed that the distribution of lamotrigine from plasma into brain tissue is only limited by blood flow, suggesting that lamotrigine crosses the blood-brain barrier by simple diffusion. The parallel decline of lamotrigine concentrations in the brain and those measured in plasma also suggests no excessive retention of the drug in the brain tissue, which is consistent with the findings of Parsons et al. (1995) when they state that the rate of lamotrigine elimination from tissues is comparable to that from plasma, with the exception of the kidney and melanin-containing tissues.

The calculated brain/plasma concentration ratios rose rapidly to an equilibrium, providing a measure of the capacity of the brain to balance with plasma and to maintain lamotrigine brain concentrations comparable to those in the circulation. The results obtained showed that the lamotrigine brain tissue concentrations are approximately twofold higher than those in plasma. Despite the different proce- dures involved, the ratio found is in accordance with those reported before (Parsons et al., 1995; Walton et al., 1996). Such a good distribution of lamotrigine in the brain is certainly a result of the basic and lipophilic properties of the molecule, which permits it to cross the blood-brain barrier easily and to have high affinity to the brain tissue.

Additionally, lamotrigine had effect in the maximal electroshock seizure test, which reflects its clinical efficacy against generalized tonic-clonic seizures (Löscher et al., 1991). The results obtained revealed that the intraperitoneal administration of $10 \mathrm{mg} / \mathrm{kg}$ of lamotrigine considerably protects rats from maximal electroshock induced-seizures in a time-range of $0.5-12-\mathrm{h}$ post-dose. This profile is coherent with a rapid onset of lamotrigine activity as well as with a long duration of action of the drug, as reported before (Miller et al., 1986). The absence of protection at 48$\mathrm{h}$ post-dose was in conformity with the low lamotrigine levels determined at that time either in the plasma or in the brain. Because no more protection was observed at $48 \mathrm{~h}$ after dosing, the study of the anticonvulsant response was ended at that time-point, although the plasma and brain sampling times were prolonged until 120 -h post-dose (when the determined brain lamotrigine levels felt below the limit of quantification of the analytical technique).

Lastly, the experimental design performed provided an opportunity for assessment of the kind of relationship established between lamotrigine plasma or brain levels and the anticonvulsant response. The results obtained revealed that the drug in the brain correlates well with the anticonvulsant response: maximal anticonvulsant activity corresponded to the highest lamotrigine brain levels achieved. Interestingly, an apparent dissociation of anticonvulsant effect from brain concentrations in the period between 12 and $24 \mathrm{~h}$ was observed. In fact, the percentage of rats protected against maximal electroshock is $62.5 \%$ at $12 \mathrm{~h}$ and it is only $12.5 \%$ at $24 \mathrm{~h}$, while over that time, the falling brain concentrations are of the order of only about $0.2 \mathrm{mg} / \mathrm{l}$ (Table 1). Unfortunately, in the absence of additional microdialysis studies, it cannot be clarified if this situation reflects differences in the ratio unbound/bound drug within the brain over that period or if it may be attributed to some sort kind of tolerance. Nevertheless, this situation does not impede the characterization of the relationship between drug in brain and anticonvulsant response as being a straight line. Relative to the effect-plasma concentration relationship, the regression analysis performed revealed that a linear relationship can only be established after rejecting the two first data points. In fact, as shown in Table 1, the relatively high plasma levels of lamotrigine found in the first sampling hour did not correspond to high lamotrigine brain levels, since the drug had not yet enough time to cross the blood-brain barrier and reach equilibrium. Therefore, taking into account the fact that lamotrigine acts in the central nervous system, the relatively high lamotrigine plasma levels found in the first sampling hour could not correspond to high maximal 
electroshock protection. Consequently, the results obtained revealed that the drug in the plasma correlates well with the anticonvulsant response only after the plasma-brain system had reached equilibrium.

In conclusion, the results obtained in this study demonstrated the existence of a linear relationship between lamotrigine in the plasma and lamotrigine in the brain (since the system has reached equilibrium) in addition to a linear relationship between lamotrigine in the brain and the anticonvulsant response obtained. So, it might be suggested that the plasma levels of lamotrigine are good indicators of the concentration of lamotrigine at its site of action and, consequently, that they are strongly correlated with the anticonvulsant effect of lamotrigine. However, since there is no clinical evidence of such a direct relationship, other justifications for the existing difficulty in the establishment of a relationship between the dose of lamotrigine administered and the clinical effect observed should be made. To some extent, the large inter-patient variability observed in dosage requirements is related to wide differences in lamotrigine pharmacokinetics, especially among patients also receiving antiepileptic drugs that inhibit or induce lamotrigine metabolism. Besides these drug interactions, the inter-patient variability may also be explained by age-dependent changes in drug disposition, disease states, seizure type or genetic factors (e.g., an over-expression of P-glycoprotein in epileptic tissue (Löscher and Potschka, 2002; Potschka et al., 2002)). So, the present results suggest further studies to a better understanding of this new generation antiepileptic drug.

\section{Acknowledgements}

We thank the Wellcome Research Laboratories for the supply of lamotrigine.

\section{References}

Adusumalli, V.E., Wichmann, J.K., Kucharczyk, N., Sofia, R.D., 1993. Distribution of the anticonvulsant felbamate to cerebrospinal fluid and brain tissue of adult and neonatal rats. Drug Metab. Dispos. 21, 1079-1085.

Bartoli, A., Guerrini, R., Belmonte, A., Alessandrì, M.G., Gatti, G., Perucca, E., 1997. The influence of dosage, age, and comedication on steady state plasma lamotrigine concentrations in epileptic children: a prospective study with preliminary assessment of correlations with clinical response. Ther. Drug Monit. 19, 252-260.

Browning, R.A., Nelson, D.K., 1985. Variation in threshold and pattern of electroshock-induced seizures in rats depending on site of stimulation. Life Sci. 37, 2205-2211.

Castel-Branco, M.M., Falcão, A.C., Macedo, T.A., Caramona, M.M., Lopez, F.G., 2001. Lamotrigine analysis in blood and brain by high-performance liquid chromatography. J. Chromatogr., B 755, 119-127.

Castel-Branco, M.M., Figueiredo, I.V., Falcão, A.C., Macedo, T.R.A., Caramona, M.M., 2002. Influence of administration vehicles and drug for- mulations on the pharmacokinetic profile of lamotrigine in rats Fundam. Clin. Pharmacol. 16, 1-6.

Deguchi, Y., Inabe, K., Tomiyasu, K., Nozawa, K., Yamada, S., Kimura, R., 1995. Study on brain interstitial fluid distribution and blood-brain barrier transport of baclofen in rats by microdialysis. Pharm. Res. 12, $1838-1844$.

Goa, K.L., Ross, S.R., Chrisp, P., 1993. Lamotrigine-a review of its pharmacological properties and clinical efficacy in Epilepsy. Drugs 46, $152-176$.

Kilpatrick, E.S., Forrest, G., Brodie, M.J., 1996. Concentration-effect and concentration-toxicity relations with lamotrigine: a prospective study. Epilepsia 37, 534-538.

Löscher, W., Potschka, H., 2002. Role of multidrug transporters in pharmacoresistance to antiepileptic drugs. J. Pharmacol. Exp. Ther. $301,7-14$.

Löscher, W., Fassbender, C.P., Nolting, B., 1991. The role of technical, biological and pharmacological factors in the laboratory evaluation of anticonvulsant drugs: II. Maximal electroshock seizure models. Epilepsy Res. 8, 79-94.

Miller, A.A., Wheatley, P., Sawyer, D.A., Baxter, M.G., Roth, B., 1986. Pharmacological studies on lamotrigine, a novel potential antiepileptic drug: I. Anticonvulsant profile in mice and rats. Epilepsia 27, 483-489.

Morris, R.G., Black, A.B., Harris, A.L., Batty, A.B., Sallustio, B.C., 1998 Lamotrigine and therapeutic drug monitoring: retrospective survey following the introduction of a routine service. Br. J. Clin. Pharmacol. 46, $547-551$.

Parsons, D.N., Dickins, M., Morley, T.J., 1995. Lamotrigine: absorption, distribution, and excretion. In: Levy, R.H., Mattson, R.H., Meldrum, B.S. (Eds.), Antiepileptic Drugs, 4th ed. Raven Press, New York, pp. $877-881$.

Perucca, E., 2002. Marketed new antiepileptic drugs: are they better than old-generation agents? Ther. Drug Monit. 24, 74-80.

Potschka, H., Fedrowitz, M., Löscher, W., 2002. P-Glycoprotein-mediated efflux of phenobarbital, lamotrigine, and felbamate at the blood-brain barrier: evidence from microdialysis experiments in rats. Neurosci. Lett. 327, 173-176.

Ramzan, I., Levy, G., 1989. Relationship between concentration and anticonvulsant effect of phenytoin against electroshock-induced seizures in rats: comparison of sampling sites for concentration determinations. J. Pharm. Sci. 78, 448-451.

Richens, A., 1994. Safety of lamotrigine. Epilepsia 35 (suppl 5), S37-S40.

Stefani, A., Spadoni, F., Siniscalchi, A., Bernardi, G., 1996. Lamotrigine inhibits $\mathrm{Ca}^{2+}$ currents in cortical neurons: functional implications. Eur. J. Pharmacol. 307, 113-116.

Swinyard, E.A., Brown, W.C., Goodman, L.S., 1952. Comparative assays of antiepileptic drugs in mice and rats. J. Pharmacol. Exp. Ther. 106, 319-330.

Waldmeier, P.C., Baumann, P.A., Wicki, P., Feldtrauer, J.J., Stierlin, C., Schmutz, M., 1995. Similar potency of carbamazepine, oxcarbazepine, and lamotrigine in inhibiting the release of glutamate and other neurotransmitters. Neurology 45, 1907-1913.

Walker, M.C., Tong, X., Perry, H., Alavijeh, M.S., Patsalos, P.N., 2000. Comparison of serum, cerebrospinal fluid and brain extracellular fluid pharmacokinetics of lamotrigine. Br. J. Pharmacol. 130, 242-248.

Walton, N.Y., Jaing, Q., Hyun, B., Treiman, D.M., 1996. Lamotrigine vs. phenytoin for treatment of status epilepticus: comparison in an experimental model. Epilepsy Res. 24, 19-28.

Wheatley, P.L., Miller, A.A., 1989. Effects of lamotrigine on electrically induced afterdischarge duration in anaesthetised rat, dog, and marmoset. Epilepsia 30, 34-40.

Yamaoka, K., Tanigawara, Y., Nakagawa, T., Uno, T., 1981. A pharmacokinetic analysis program (MULTI) for microcomputers. J. Pharmacobiodyn. $4,879-885$. 